\title{
The challenges of implementing low-dose computed tomography for lung cancer screening in low- and middle-income countries
}

\author{
Eduardo Edelman Saul ${ }^{1 凶}$, Raquel B. Guerra², Michelle Edelman Saul', Laercio Lopes da Silva ${ }^{4}$, \\ Gabriel F. P. Aleixo ${ }^{5}$, Raquel M. K. Matuda ${ }^{6}$ and Gilberto Lopes ${ }^{7}$
}

Lung cancer accounts for an alarming human and economic burden in low- and middle-income countries (LMICs). Recent landmark trials from high-income countries (HICs) by demonstrating that low-dose computed tomography (LDCT) screening effectively reduces lung cancer mortality have engendered enthusiasm for this approach. Here we examine the effectiveness and affordability of LDCT screening from the viewpoint of LMICs. We consider resource-restricted perspectives and discuss implementation challenges and strategies to enhance the feasibility and cost-effectiveness of LDCT screening in LMICs.

ung cancer is the most frequently diagnosed malignancy and the leading cause of cancer death worldwide: GLOBOCAN estimated 2,093,876 new cases and a total of 1,761,007 deaths in 2018 , with $61 \%$ of incidence and $65.3 \%$ of mortality occurring in LMICs ${ }^{1}$. The human and economic burden of this cancer type in resource-scarce populations is an urgent public health crisis with an expected upward trend, given the steadily increasing prevalence of smoking and air pollution in those countries ${ }^{2}$.

Non-small-cell lung cancer (NSCLC) accounts for approximately $85 \%$ of all lung cancers ${ }^{1}$. Related symptoms are nonspecific, usually manifested as lasting cough and dyspnea ${ }^{3}$. Roughly $75 \%$ of the cases present when the disease is locally advanced (stage IIIC) or metastatic (stage IV), when it has 5-year survival rates of $13 \%$ and $0 \%$, respectively. In contrast, when detected at stage IA, usually in asymptomatic individuals, NSCLC can be treated with curative intent, with overall survival of up to $92 \%$ (ref. ${ }^{4}$ ). These stark differences provide the opportunity to improve survival in the high-risk population by early detection through lung cancer screening (LCS). However, chest X-ray (CXR) with and without sputum cytology as a screening strategy has failed to demonstrate benefit for lung cancer mortality ${ }^{5,6}$. Renewed enthusiasm arose when the National Lung Screening Trial (NLST) showed a $20 \%$ mortality reduction in high-risk individuals using LDCT screening ${ }^{7}$, which was recently buoyed by results of the Dutch-Belgian Lung Cancer Screening Trial $(\mathrm{NELSON})^{8}$

LCS programs require the availability of a complex healthcare infrastructure and considerable economic resources, making LDCT screening efficacy and affordability highly variable ${ }^{2}$, especially in emerging economies with fragmented and underfinanced health systems. Herein, we present the LCS development and LMIC perspective of lung cancer epidemiology, discussing cost-effectiveness and strategies for LDCT screening implementation in the daily LMIC oncology practice.

\section{LCS development}

The first study to assess LCS, in the 1960s, evaluated 55,034 men $\geq 40$ years of age and compared CXR results every 6 months for 3 years, with a control group receiving CXR at the beginning and end of the study ${ }^{9}$. Subsequently, a cooperative study at three different US sites, including over 30,000 male smokers, evaluated dual-screening (CXR plus sputum cytology) versus control groups undergoing CXR or no intervention ${ }^{6}$. In Czechoslovakia, semiannual or 3-year-interval dual screening was compared with no screening, including high-risk men aged 40-64 years with lifetime cigarette consumption of $\geq 150,000$ (ref. ${ }^{5}$ ). Finally, the Prostate, Lung, Colorectal, and Ovarian Cancer (PLCO) Screening Trial randomized 154,091 participants 55-74 years of age to annual CXR versus standard care, substantiating the lack of mortality benefit of these strategies ${ }^{10}$.

The Early Lung Cancer Action Program (ELCAP), initiated in 1992, was the first to gauge the use of LDCT for LCS. One thousand participants with a cigarette smoking history of $\geq 10$ packs per year underwent baseline ${ }^{11}$ and annual (841 individuals) ${ }^{12}$ LDCT and CXR, demonstrating an increase in early-stage diagnosis rate with LDCT. In 2006, the follow-up International Early Lung Cancer Action Program (I-ELCAP) study ${ }^{13}$ included 31,567 individuals from Japan, the United States, Europe, Israel and China, with participants $>40$ years old and subject to high-risk factors such as cigarette smoking, occupational exposure (asbestos, beryllium, uranium or radon) or second-hand smoke exposure. This study reported an $85 \%$ screening detection rate of stage I lung cancer, with $80 \%$ estimated overall 10-year survival, which increased to $92 \%$ if resection occurred within 1 month of diagnosis. Likewise, observational studies ultimately suggested that screening with LDCT could potentially detect curable lung cancer ${ }^{14-19}$. This growing body of evidence encouraged further confirmatory randomized controlled trials $(\mathrm{RCTs})^{20}$.

'Department of Medicine, Jackson Memorial Hospital/University of Miami, Miami, FL, USA. ${ }^{2}$ Centro Paulista de Oncologia-Oncoclinicas Group, São Paulo, Brazil. ${ }^{3}$ Department of Medicine, Universidade Estácio de Sá, Rio de Janeiro, Brazil. ${ }^{4}$ Department of Medicine, Metrowest Medical Center/Tufts University School of Medicine, Framingham, MA, USA. ${ }^{5}$ Department of Hematology-Oncology, University of North Carolina at Chapel Hill, Chapel Hill, NC, USA. ${ }^{6}$ Division of Oncology, Oncoclinicas Group, Brasilia, Brazil. ${ }^{7}$ Division of Hematology-Oncology, Department of Medicine, Miller School of Medicine, Sylvester Comprehensive Cancer Center, University of Miami, Miami, FL, USA. ${ }^{\star}$ e-mail: eduardo.saul@jhsmiami.org 
The landmark NLST, in 2011, was the first to demonstrate mortality reduction with LDCT screening. This trial randomized 53,454 high-risk participants to undergo three annual screenings with LDCT versus CXR. Eligible participants were current or former smokers 55-74 years of age with a $\geq 30$-pack-per-year smoking history and $<15$ years since quitting. Cases were detected at either stage IA or IB in $63 \%$ of patients in the screening arm, characterizing stage migration. A reduction of $20 \%$ and $6.7 \%$ in lung cancer and all-cause relative mortality, respectively, was observed ${ }^{7}$.

After a sequence of RCTs with limited power raised uncertainties by publishing inconclusive and heterogeneous results ${ }^{21-26}$, the NELSON trial ${ }^{8}$, the only fully powered RCT following the NLST, assuaged doubts about the potential mortality benefit of LDCT screening ${ }^{27}$. The NELSON trial randomized 15,792 current or former smokers 50-75 years of age, with smoking history of $>15$ cigarettes daily for $>25$ years or $>10$ cigarettes daily for $>30$ years and $\leq 10$ years after quitting, to four rounds of LDCT at baseline and 1 -, 2- and 2.5-year intervals versus no screening. Results showed that $58.6 \%$ of the screening-detected cases were in the early stages (IA and IB). At the 10-year follow-up, the reduction in lung cancer mortality was $24 \%$ and $33 \%$ among men and a small sample of women, respectively. The trial was not powered to show possible favorable all-cause mortality difference (expected at $2.5 \%$ ).

Another recent RCT, the Multicentric Italian Lung Detection trial ${ }^{28}$, provided additional evidence that prolonged intervention beyond five years could enhance screening benefits. It enrolled 4,099 participants aged 49-75 years, with a $\geq 20$-pack-per-year smoking history and who were current smokers or had quit $<10$ years prior, for annual or biennial LDCT screening versus no intervention. Results demonstrated 39\% risk reduction (RR) for 10-year lung cancer mortality, and improved lung cancer and all-cause mortalities beyond the fifth year of screening, with RRs of $58 \%$ and $32 \%$, respectively.

\section{Current landscape in LMICs}

In the following sections we discuss the current landscape of lung cancer epidemiology and screening in LMICs.

Lung cancer risk factors and epidemiology in LMICs. Smoking is the critical risk factor credited to causing $85 \%$ of all lung cancers in current or past smokers, with a direct correlation between risk and smoking load ${ }^{29}$. Over $80 \%$ of the 1.3 billion smokers worldwide reside in $\mathrm{LMICs}^{2}$, accounting for more than $70 \%$ of all global smoking-related deaths ${ }^{30}$. The increased smoking prevalence that is associated with changes in tobacco habits and expanding tobacco markets, combined with the often large populations of LMICs, is generating profound public health challenges, with substantial consequences expected in the decades to follow ${ }^{2,31}$.

Although lung cancer incidence is higher in male smokers, there is an upward trend in women with no history of cigarette consumption $^{32}$. In fact, $10-25 \%$ of lung cancer occurs in those who have never smoked, with a relatively high prevalence in Eastern Asian women ${ }^{33}$. Indoor pollution from burning charcoal for heating and cooking, second-hand smoke and outdoor air pollution are the main risk factors for this phenomenon ${ }^{34}$. The situation is especially aggravated in China, where cigarette smoking is responsible for approximately $57.5 \%$ of lung cancer in men, but only $11.5 \%$ in women ${ }^{35}$, whereas in the United States it is estimated to influence $84.4 \%$ and $78.9 \%$ cases, respectively ${ }^{36}$.

In 2018, upper-middle-income countries (UMICs) had the highest lung cancer incidence and mortality among LMICs, with $1,032,425$ cases and 924,483 deaths ${ }^{1}$. Rates continue to grow, with over 2 million lung-cancer-related deaths expected in LMICs by 2040, contrasting with about 850,000 in HICs (Fig. 1). More specifically, lung cancer incidence in China has increased $465 \%$ over the past 30 years, ranking the highest among all cancer types since the beginning of this century, and accounting for more than one-third of global lung cancer mortality ${ }^{37}$. Gross domestic product (GDP) correlates negatively with case fatality, being estimated at $90 \%$ versus $74.8 \%$ in LMICs and HICs, respectively ${ }^{38}$. This phenomenon is multifactorial and likely associated with challenges in access to diagnostic tools leading to delayed detection, as well as barriers to adequate treatment ${ }^{39}$.

LCS in LMICs. Although evidence for the efficacy of LDCT screening in LMICs is growing, it is still scarce and indeterminate ${ }^{40}$. Furthermore, LCS has been placed under scrutiny in light of the potential harms, primarily including false-positive rates, incidental findings, diagnostic procedure complications and overdiagnosis ${ }^{20}$. Results are heterogeneous across RCTs in HICs and may differ from real-life experience in LMICs. Hence, generalizing the HIC experience may be misleading, making context- and country-specific studies a necessity.

Ongoing and finalized LDCT screening studies in LMICs (Table 1) have been concentrated in UMICs. A large Indian cohort supported the efficacy of LDCT upon screening 28,351 and 25,356 individuals with baseline and repeat LDCT, respectively. The researchers detected 480 cases, $84 \%$ at stage I. Results were equivalent to previous HIC data, indicating 10 -year survival rates estimated at $87 \%$ in stage I cases and $91 \%$ in those resected within 1 month from diagnosis ${ }^{41}$.

In China, LDCT screening trials started in 1994 in Zhuhai with an empirical protocol of 2-year follow-up for baseline CT scans, later becoming the first I-ELCAP site in that country in 2003. The incorporation of the international protocol and training resulted in a shorter interval between lung cancer detection and surgery (213 versus 96 days), with an improvement in 5-year survival (75\% versus $95 \%$, respectively ${ }^{42}$. In 2009, LDCT screening was included within the Chinese Central Government Public Health Special Subsidy Program, leading to an ongoing prospective multicenter observational study evaluating the effectiveness and feasibility of LDCT screening implementation in that country ${ }^{43}$. By 2015, the program had expanded to ten centers, with early-detection rates of $40 \%$ and $56 \%$ in baseline and annual screening, respectively, which is much higher than the Chinese usual care rate of $7 \%$ (ref. ${ }^{44}$ ). The Cancer Screening Project in Urban Areas of China started in 2012 and was designed to offer 210,000 cost-free baseline LDCT screenings until 2017 (ref. ${ }^{45}$ ). Both programs included health promotion to increase adherence, training for local physicians and technicians, and smoking cessation interventions.

In 2018, a systematic review found 23 prospective cohort studies and RCTs evaluating LCS in China, with the majority reporting preliminary results and suggesting a benefit from LDCT screening $^{46}$. Moreover, a simulation model analyzing data from 100,000 Chinese smokers $45-80$ years of age indicated that LDCT screening would reduce mortality between $40.2 \%$ and $5.4 \%$ in the best- and worst-case scenarios, respectively ${ }^{47}$. Given the accumulation of local evidence, experts released a consensus-based China National Lung Cancer LDCT Screening Guideline in 2015 and an updated version in 2018 (ref. ${ }^{48}$ ).

Data from a parallel retrospective analysis of the ongoing Moscow Lung Cancer Screening trial showed that concurrent screening detection of findings associated with chronic obstructive pulmonary disease (COPD) and cardiovascular disease could improve LDCT screening diagnostic value ${ }^{49}$. Another current collaborative multicenter study, the Netherlands-China Big-3 screening, will also focus on lung cancer, COPD and cardiovascular disease detection in both countries ${ }^{50}$.

Concerning false positives, the Brazilian Lung Cancer Screening Trial $^{51}$, performed between 2013 and 2014, enrolled 790 participants according to the NLST inclusion criteria and used the same positive nodule threshold of $>4 \mathrm{~mm}$. The prevalence of lung cancer was 


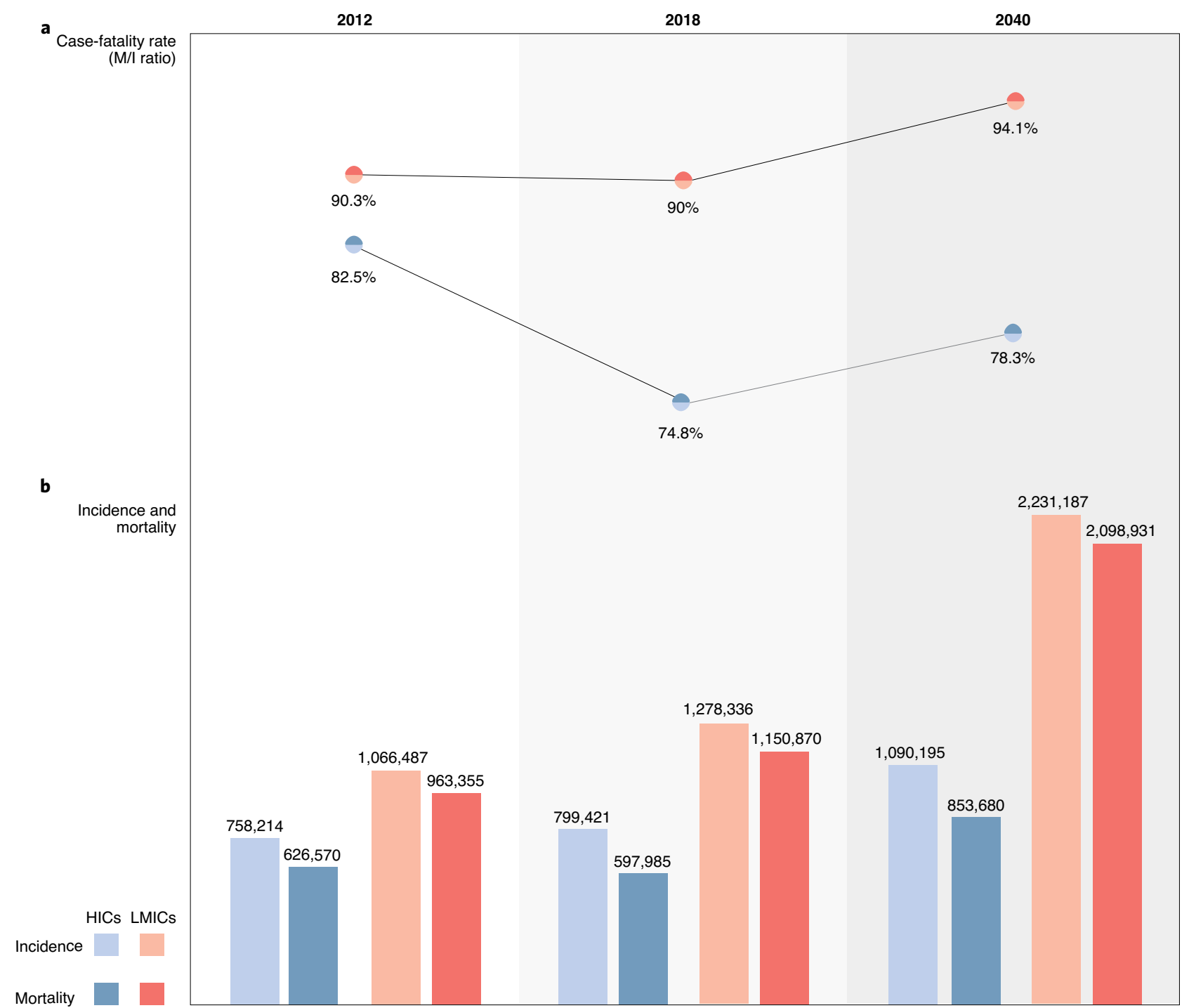

Fig. 1 | The growing disparities in lung cancer incidence, mortality and case fatality between LMICs and HICs over time. $\mathbf{a}$, $\mathbf{b}$, The disparities in case-fatality rates (mortality/incidence (M/I) ratio) (a) and disproportionate uptrends in lung cancer incidence and mortality (b) between LMICs and HICs from 2012 to the 2040 estimated values. These phenomena are likely to be multifactorial and a consequence of increasing smoking prevalence, the often large populations in LMICs, challenges in access to diagnostic tools leading to delayed detection, and barriers to adequate treatment. Methods: incidence and mortality values from 2012 and 2018 were extracted from ref. ${ }^{1}$ and used to calculate case-fatality. The 2040 values were calculated for each group (LMICs and HICs) according to the World Bank's country income classification for 2019-2020 (ref. ${ }^{88}$ ) and based on the estimates available by country in ref. ${ }^{1}$ (see Supplementary Tables 1 and 2 ).

similar to that in the NLST (1.3\%), with $80 \%$ of cases diagnosed at stage IA or IB. A higher rate of positive imaging (39.6\% vs. $26 \%$ ) was likely associated with the high tuberculosis (TB) prevalence in Brazil, resulting in a lower positive predictive value. Despite the increased number of control follow-up LDCTs, the number of invasive procedures and lung cancer diagnoses were similar to those in other reports, suggesting that LDCT screening is applicable in populations with high TB prevalence.

In Thailand, another TB-endemic area, a nonrandomized prospective study evaluated LDCT screening in 634 smokers aged 50-70 years. Preliminary results released after three rounds demonstrated screening-detected lung cancer in $1.4 \%$ of participants, with $56 \%$ of cases at stage I and a positive predictive value of $23 \%$. The incidence of active TB was $0.50 \%$ and $0.52 \%$ at first- and second-year rounds, respectively. The authors suggested that a high prevalence of pulmonary nodules challenged diagnosing and staging lung cancer with screening, and proposed a nodule management protocol adapted to TB-endemic settings ${ }^{52}$. Accordingly, the Southern Africa Thoracic Society guidelines, published in 2019, endorsed a conservative cutoff for positive nodules considering the high local TB prevalence $^{53}$. In Table 2, we compare the main US recommendations with the current LMIC guidelines ${ }^{54,55}$.

\section{Screening delivery in LMICs}

Screening approaches are defined as opportunistic or organized ${ }^{2}$. The first occurs at the individual level, whereas the second entails a population-based program with delivery of screening as part of a screening-to-treatment continuum of care $^{56}$. Access to healthcare, identification of high-risk patients, screening uptake, adherence and implementation, followed by the interpretation and communication of results and timely referral for further testing and treatment, are the fundamental steps of organized screening programs ${ }^{57}$. In the following sections, we outline these steps and provide guidance to overcome hindrances shared by various LMICs. 
Table 1 | Ongoing lung cancer screening trials in LMICs

\begin{tabular}{|c|c|}
\hline Country & Study title \\
\hline China & $\begin{array}{l}\text { Community-based Early } \\
\text { Stage Lung Cancer Screening } \\
\text { With Low-dose Computed } \\
\text { Tomography in China }\end{array}$ \\
\hline China & $\begin{array}{l}\text { Community-based Lung Cancer } \\
\text { Screening With Low-dose CT in } \\
\text { China (CLUS Study) Version } 2.0\end{array}$ \\
\hline China & $\begin{array}{l}\text { Methods of Computed } \\
\text { Tomography Screening and } \\
\text { Management of Lung Cancer } \\
\text { in Tianjin: A Population-based } \\
\text { Cohort Study }\end{array}$ \\
\hline China & $\begin{array}{l}\text { Screening and Diagnosing for } \\
\text { Early Lung Cancer in Shanghai } \\
\text { Communities With Imaging } \\
\text { Procedures }\end{array}$ \\
\hline
\end{tabular}

Sample Intervention
size $^{\mathrm{a}}$

Eligibility criteria

Key Technology in Precision Diagnosis and Therapy for Early Stage Lung Cancer: a Single Arm Clinical Trial

China

Computed Tomography

Screening for Early Lung Cancer, COPD and Cardiovascular Disease in Shanghai: a Population-based Comparative Study

China Design of Self-evaluation Score Questionnaire for High-risk Groups of Lung Cancer and the Efficacy of DR Combined with LDCT for Lung Cancer Screening

China China National Cancer Early Screening Trial: Lung and Colorectal Cancer assessment and management

$\begin{array}{cl}\text { China } & \text { Low-dose Computed } \\ & \text { Tomography Screening for Lung } \\ & \text { Cancer in Relatives With Family } \\ & \text { History of Lung Cancer } \\ \text { China } & \begin{array}{l}\text { Low-dose spiral CT combined } \\ \text { with inflammatory cytokines } \\ \text { in lung cancer screening: a } \\ \text { randomized controlled trial }\end{array}\end{array}$

Age 50-75 years with: 30-pack-year smoking history or quit for 5 years; or nonsmoking women with SHS from family members who live together or co-workers in the same room with a smoking history of 30 pack-years and a subsequent smoking cessation of 5 years.

6,000 Active arm: baseline +2

biennial repeated LDCT; passive arm: baseline +2 biennial repeated questionnaire inquiries

6,000

Baseline +2 biennial repeated LDCT

\section{4,000 \\ Annual LDCT (two rounds)}

Baseline +2 rounds of biennial repeated LDCT screening

LDCT screening with visual of imaging biomarkers

A: 30;

B: 400 ;

C: 2,000 ;

D: 8,000 D: QS about RF of lung

A: 28,800; A: annual LDCT (5 rounds); B: 43,200; B: biennial LDCT (3 rounds);

C: 43,200 C: inform participants about
A: QS about RF of lung cancer patients; B, C: QS about RF of lung cancer patients and healthy volunteers; cancer patients and healthy volunteers + DR and LDCT high risks of lung cancer and recommend them to undertake lung cancer screening in other regular medical institutions

Lung cancer patient or health volunteer, age $20-90$ years, who took annual physical examinations in the West China Hospital of Sichuan University between 2007 and 2009.

Age 50-74 years, residence $>5$ years at screening site, smoking history $\geq 30$ pack-years with quit $<15$ years; or COPD, or a history of diffuse interstitial pulmonary fibrosis; or occupational exposure history $\geq 5$ years; or non-smoking women with SHS; or TB patients cured for $\geq 2$ years.

Relative of lung cancer patient(s); age $\geq 55$ years or $\geq$ the age of onset of lung cancer in proband if the family members were $<55$ years old.

LDCT; LDCT + inflammatory cytokines
Status

Ongoing

Preliminary

result

published

Ongoing smoking history or quit $<15$ years; personal exposure to carcinogens; long-term exposure district of Tianjin city for at least 3 years; lack of self-reported history of any malignant

Ongoing

Unknown history or quit $<15$ years; or age $\geq 50$ years (occupational or residential radon exposure, cancer history, family history of lung cancer, one additional risk factor.

Unknown

Unknown

Ongoing

Unknown

Unknown 
Table 1 | Ongoing lung cancer screening trials in LMICs (Continued)

\begin{tabular}{|c|c|c|c|c|c|}
\hline Country & Study title & $\begin{array}{l}\text { Sample } \\
\text { size }^{\mathrm{a}}\end{array}$ & Intervention & Eligibility criteria & Status \\
\hline India & $\begin{array}{l}\text { Utility of LDCT in Lung Cancer } \\
\text { Screening in a TB Endemic } \\
\text { Region }\end{array}$ & 200 & LDCT (single round) & $\begin{array}{l}\text { Age } 55-74 \text { years with } \geq 30 \text {-pack-year history } \\
\text { of smoking (or smoking index } \geq 600 \text { ), either } \\
\text { current smoker or quit }<15 \text { years; or age } \\
50 \text { - } 74 \text { years with } \geq 20 \text {-pack-year history of } \\
\text { smoking (or smoking index } \geq 400 \text { ), either } \\
\text { current or former smoker, with COPD } \\
\text { or family history of lung cancer in any } \\
\text { first-degree relative. }\end{array}$ & Ongoing \\
\hline Russia & $\begin{array}{l}\text { CT Screening For Lung Cancer in } \\
\text { High Risk Patients: the Russian } \\
\text { Study }\end{array}$ & 369 & Baseline LDCT & $\begin{array}{l}\text { Age } 55-75 \text { years with } \geq 30 \text {-pack-year smoking } \\
\text { history and quit } \leq 10 \text { years. }\end{array}$ & $\begin{array}{l}\text { Completed; } \\
\text { not published }\end{array}$ \\
\hline
\end{tabular}

Results were obtained by a search of the WHO International Clinical Trials Registry Platform (http://apps.who.int/trialsearch/default.aspx) and National Institute of Health US National Library of Medicine (https://clinicaltrials.gov/), accessed 4 March 2020, with the search term 'lung cancer screening'; studies evaluating LDCT lung cancer screening in LMICs were included. DR, digital radiography; QS, questionnaire survey; RF, risk factors; SHS, second-hand smoking. ${ }^{\mathrm{a}} \mathrm{A}, \mathrm{B}, \mathrm{C}, \mathrm{D}$, study arms.

Table 2 | Comparison of US versus LMIC main guidelines

\begin{tabular}{|c|c|c|c|c|c|c|c|}
\hline \multirow[t]{2}{*}{ Guidelines } & \multirow[t]{2}{*}{ Year } & \multirow[b]{2}{*}{$\begin{array}{l}\text { Age } \\
\text { (years) }\end{array}$} & \multicolumn{3}{|c|}{ Eligible population } & \multirow[t]{2}{*}{ Recommendation } & \multirow{2}{*}{$\begin{array}{l}\text { Positive } \\
\text { nodule } \\
\text { cutoffs }\end{array}$} \\
\hline & & & Pack-years & Quit-years & Considerations & & \\
\hline \multicolumn{8}{|l|}{ US } \\
\hline \multirow[t]{2}{*}{$\begin{array}{l}\text { National Comprehensive } \\
\text { Cancer Network (NCCN) }\end{array}$} & 2020 & $55-77$ & $\geq 30$ & $\leq 14$ & $\begin{array}{l}\text { Discontinue if no longer } \\
\text { candidate for definitive } \\
\text { treatment }\end{array}$ & Annual LDCT & $>5 \mathrm{~mm}$ \\
\hline & & $\geq 50$ & $\geq 20$ & $N / A$ & Other risk factors ${ }^{a}$ & & \\
\hline $\begin{array}{l}\text { Screening for Lung Cancer: US } \\
\text { Preventive Services Task Force } \\
\text { Recommendation Statement }{ }^{\mathrm{b}}\end{array}$ & 2014 & $55-80$ & $\geq 30$ & $\leq 15$ & $\begin{array}{l}\text { Discontinue if: } \\
\text { Limited life expectancy } \\
\text { Lack of ability or willingness } \\
\text { for curative treatment }\end{array}$ & Annual LDCT & $>5 \mathrm{~mm}$ \\
\hline \multicolumn{8}{|l|}{ LMICs } \\
\hline $\begin{array}{l}\text { Recommendations for lung } \\
\text { cancer screening in Southern } \\
\text { Africa }\end{array}$ & 2019 & $55-74$ & $\geq 30$ & $\leq 15$ & $\begin{array}{l}\text { Discontinue if: } \\
\text { Limited life expectancy } \\
\text { Lack of ability or willingness } \\
\text { for surgery. }\end{array}$ & Annual LDCT & $\geq 6 \mathrm{~mm}$ \\
\hline $\begin{array}{l}\text { China National Lung Cancer } \\
\text { Screening Guideline }\end{array}$ & 2018 & $50-74$ & $\geq 20$ & $\leq 5$ & $\begin{array}{l}\text { No history of lung cancer } \\
\text { General good health } \\
\text { Fit for surgery and willing to } \\
\text { further investigate }\end{array}$ & Annual LDCT & $\begin{array}{l}\geq 5 \mathrm{~mm} \text { or } \\
\mathrm{NCN}\end{array}$ \\
\hline
\end{tabular}

Sources: refs. ${ }^{88,53-55}$. N/A, not applicable; NCN, noncalcified nodule. ${ }^{a}$ Tobacco smoking; contact with radon, asbestos or other cancer-causing agents; history of cancer, family history of lung cancer; history of COPD or pulmonary fibrosis. ${ }^{\circ}$ The US Preventive Services Task Force Recommendation Statement draft released on 7 July 2020 proposed an updated and broadened eligibility criteria, recommending annual screening for lung cancer with LDCT in adults 50-80 years of age who have a 20-pack-year smoking history and currently smoke or have quit within the past 15 years.

Barriers and solutions for LCS access and patient identification and adherence. Structural and regional hurdles play a critical role in access to screening in LMICs. Lack of transportation, long travel distances and poor road conditions can make healthcare practically inaccessible, especially in rural areas ${ }^{58}$. In rural western China, $47 \%$ of impoverished people live within $2 \mathrm{~km}$ of a medical clinic, compared with $65 \%$ in metropolitan areas ${ }^{59}$. Hence, in addition to building infrastructure that equitably reaches the population in the long term, the employment of mobile CT scanners and one-stop 'lung health-checks' among community centers ${ }^{60}$, as well as the provision of complementary transportation within screening programs, are essential strategies to address such scenarios ${ }^{61}$.

Moreover, physician unawareness of screening guidelines and lack of time for counseling represent challenges for the identification and adherence of eligible high-risk individuals in $\mathrm{LMICs}^{62,63}$. For instance, in Egypt, a national pilot survey demonstrated that physicians do not frequently perform LCS, notwithstanding expert group recommendations ${ }^{64}$. In Pakistan, a sample survey of 200 primary care physicians revealed that even though $54.5 \%$ acknowledged the effectiveness of LCS, only $33.3 \%$ reported ordering the test $^{63}$. Furthermore, counseling has a significant impact on screening adherence, as corroborated by a study among Korean men showing that physician discussions with patients regarding the harms and benefits of LDCT screening improved participation from $10.6 \%$ to $95.1 \%$ (ref. ${ }^{65}$ ). Therefore, an optimized and affordable provider education program should be a priority in early implementation phases $^{62}$. In HICs, the use of electronic health records to help identify high-risk individuals also enhanced referral rates, but such a tool is not readily available in most LMIC clinics ${ }^{66}$. Employing community health workers (CHWs) for screening recruitment and counseling has been successful in LMICs ${ }^{67}$ and might be a feasible and practical aid to busy clinicians ${ }^{68}$. 
Cultural barriers, including fear, stigmas and blame for patients with lung cancer, also affect screening adherence, as has been demonstrated in several communities ${ }^{69}$. In Pakistan, a survey among 186 smokers demonstrated that fear of positive results (52.6\%) and skepticism regarding treatment effectiveness (47.4\%) were significant reasons for lack of screening participation ${ }^{63}$. In India, $53 \%$ of 500 survey respondents believed that cancer patients were to blame for presenting with the disease ${ }^{70}$. Other unfounded beliefs, such as that death is inevitable when cancer is present ('cancer fatalism') ${ }^{59}$ or that 'cancer is contagious', and the fact that cancer is frequently a taboo topic ${ }^{71}$, particularly in populations of low socioeconomic status, contribute to a misinformed public that is distrustful of the healthcare system and thus unfortunately subject to delayed diagnosis and worse outcomes ${ }^{59}$.

Social disparities, such as extreme poverty and gender discrimination, also jeopardize equitable access to screening and treatment ${ }^{2}$. Notably, in India, people from disadvantaged castes, with less education or with lower income face increased risks of dying from cancer $^{72}$. Mortality rates are also higher in women from rural populations when compared with urban residents ${ }^{72}$. Reports show that $40 \%$ of Indian women would be willing to participate in a screening program but were unable due to socially imposed domestic chores ${ }^{73}$.

Therefore, understanding and developing culture-sensitive screening approaches is essential for the realization of LCS programs in $\mathrm{LMICs}^{74}$. As noted above, integrating CHWs into existing healthcare systems can serve to navigate patients through screening options and processes. Importantly, CHWs would be best positioned to understand the distinctive cultural context in which patients conduct their lives. For example, CHWs could triage eligible cases; promote smoking cessation activities and follow-up, thereby increasing patient adherence; help reduce stigma by answering culturally pertinent questions; and ultimately catalyze screening access and care completion. In India, a cluster-randomized trial demonstrated that CHWs identified women eligible for screening and increased their compliance with at least one round of screening for breast and cervical cancer to $94 \%$ and $84 \%$, respectively ${ }^{75}$. Importantly, the decision to participate in the screening program must follow from an informed discussion about benefits and potential harms, and a shared commitment between physician and patients to pursuing further investigation when appropriate ${ }^{76}$.

The LDCT screening-to-treatment continuum in LMICs. Once a population-based program is planned, a rise in demand for CT scans, either for screening or as follow-up, is expected. Thus, it is vital to have equipment utility available and strategically distributed across the territory. However, building infrastructure is costly and time-consuming and may be unaffordable in many LMICs. Currently, there is a disproportion between in LMICs versus HICs in CT scanners available ${ }^{77}$.

Furthermore, the management of screening-detected nodules is a complex task that requires experienced radiologists to perform standardized interpretation of the images. Training with specific protocols such as the American College of Radiology protocol (Lung-RADS $)^{78}$ or using the updated I-ELCAP volumetric-based nodule measurement guidelines ${ }^{79}$ can mitigate overdiagnosis and false-positive results and, in particular, intra-observer variability ${ }^{80}$. Adopting such protocols can also provide a simplified workflow for assessing positive findings and decrease the number of unnecessary follow-up CT scans, thus incurring less patient anxiety and lower $\operatorname{costs}^{8,27}$. Local nodule management guidelines are currently under validation in Asia $^{44,81}$, Brazil ${ }^{51}$ and South Africa ${ }^{53}$, considering diversities in local lung cancer epidemiology and aiming, in particular, to reduce false-positives in TB-endemic areas.

Diagnosis confirmation and treatment require a multidisciplinary team of experts, including thoracic surgeons, interventional radiologists, pulmonologists, oncologists and pathologists. Surgical excision, CT-guided biopsy and bronchoscopy are procedures that demand expertise and specific supplies. Immunohistochemical processing of samples for lung cancer subtyping and molecular diagnosis is essential for adequately evaluating the biopsied material. Finally, correct and timely treatment is fundamental and may involve surgery, radiotherapy, chemotherapy, immune and targeted therapies, or therapy combinations depending on disease type and stage. Though the majority of patients in LMICs have access to a certain level of cancer care, the broad availability of surgery and radiotherapy services is still an overriding concern ${ }^{82}$. In addition, apart from a privileged minority, patients in LMICs do not have access to the newest and expensive drugs that are standard of care in $\mathrm{HICs}^{83}$.

The lack of skilled human capital in some LMICs is worrisome. In India, there is 1 oncologist per 16,000 patients, contrasting with 1 per 100 in the United States ${ }^{84}$. The limited number of qualified providers is also a challenge to equitable intra-country healthcare access $^{58}$. For instance, there are 8.5 physicians or nurses per 1,000 people in urban Eastern China, compared with 2.8 per 1,000 in the rural areas ${ }^{59}$. Regardless of the method deemed complete in a country-specific context, all components of care need to be available for patients once lung cancer is detected through screening, given that incomplete treatment and limited downstream resources may cause unacceptable harm with no benefit.

LCS program quality. Monitoring and quality assessment should be central and part of an iterative process of population-based screening program improvement. Information systems, including hardware, data management software and patient intake processes, are necessary. Experience from HICs supports the practice of creating multidisciplinary teams that should include data system experts and personnel to manage and assess the program ${ }^{85}$.

Overall, population-based LCS programs should be organized within a multilevel context ${ }^{86}$. The readiness of each LMIC's healthcare system for screening program implementation must be carefully considered. National planning committees should be created with interest in the local and context-specific discussion of evidence, lung cancer epidemiology, population acceptance of screening intervention, infrastructure and availability of provider teams with technical expertise, sociopolitical milieu and costs ${ }^{87}$.

\section{Lung cancer costs and cost-effectiveness of LDCT screening in LMICs}

The World Bank's definition of LMICs covers a wide range of nations at various stages of economic and health system development $^{88}$. It comprises regions where communicable diseases such as HIV/AIDS and malaria are still pressing public health problems (for example, sub-Saharan Africa) ${ }^{89}$ and countries that currently face extremely fragile situations with uninterrupted armed conflicts and no functioning healthcare system, such as Afghanistan ${ }^{90}$. For countries experiencing such challenges, investing in LCS is not a current priority ${ }^{91}$. However, LMICs also comprise countries that are experiencing rapid economic growth, especially the UMICs (for example, China and Brazil), with populations facing a rising prevalence of non-communicable diseases ${ }^{92,93}$.

Consequently, the financial burden of cancer intensifies rapidly, with new lung cancer cases accounting for the highest costs among all cancer sites ${ }^{94}$. For instance, the China Statistical Yearbook estimated that the total inpatient costs associated with lung cancer increased from US $\$ 2.16$ billion to US $\$ 6.33$ billion between 1999 and 2005 (ref. $\left.{ }^{43}\right)$. Data from the Union's Court of Auditors in Brazil indicate that the national cost of cancer treatment has increased by $137 \%$ (from US $\$ 0.25$ billion to US $\$ 0.60$ billion) between 2002 and 2010, about three quarters of which is due to chemotherapy drug expenditure alone $^{95}$. New lung cancer therapies are remarkably costly worldwide and could undermine the health systems of LMICs. In Argentina, 
Brazil and Peru, use of immune-checkpoint inhibitors is currently cost-prohibitive ${ }^{95}$. In 2009, the Mexican National Cancer Institute estimated NSCLC costs ranging from US\$13.456 to US\$144.555 per patient diagnosed at stage I and stage IV, respectively ${ }^{96}$. Indeed, there is a direct relationship between advanced disease and higher expenses, suggesting that non-curative treatment costs may be higher than the cost of implementing screening itself ${ }^{97}$.

With more LMICs achieving publicly funded universal health coverage (UHC) - 22 countries by 2014 (ref. ${ }^{98}$ ) - there is a rising demand for high-quality healthcare delivery and infrastructure development that unfolds the challenge of resource allocation and priority-setting. Stakeholders should consider investing in cost-effective cancer control strategies, including prevention and early detection ${ }^{98}$, potentially engendering enthusiasm to evaluate the local applicability and feasibility of LDCT screening. In perspective, data from HICs indicate favorable results in comparing lung with breast and cervical cancer screening, two cancer types previously established as health priorities in $\mathrm{LMICs}^{99}$. For example, the number needed to screen (NNS) to prevent one death from lung cancer in the NLST was 320 ( ref. $^{7}$ ), compared with 2,000 for breast cancer screening mammography ${ }^{100}$. The US Food and Drug Administration reports that around 39 million mammograms are performed annually (not exclusively for screening), in contrast to 6.8 million eligible for LDCT screening ${ }^{101,102}$. Cost per life-year saved is also higher in breast, cervical and colorectal screening than in lung cancer screening ${ }^{103,104}$.

Using cost-effectiveness analysis (CEA) is crucial in such public health decision-making processes as it helps countries attain and sustain $\mathrm{UHC}^{105}$. CEA should be designed to inform stakeholders about the context-specific impact of new investment in constrained-resource settings, estimating the gap between the need and availability of required resources as well as the opportunity costs of diverting healthcare means from other potential interventions ${ }^{106}$.

Determining the appropriate economic evaluation methods is particularly difficult in LMICs, where reliable data, research capacity and funding are limited ${ }^{107}$. According to the World Health Organization (WHO) thresholds, an incremental cost-effectiveness ratio (ICER) lower than 1 GDP per capita is considered as highly cost-effective, and an ICER between 1 and 3 GDP per capita as cost-effective $^{108}$. However, there is a current argument against an ever-growing 'one-size-fits-all' threshold, and initiatives such as REVISE 2020 (ref. ${ }^{109}$ ) and the International Decision Support Initiative ${ }^{106}$ launched tailored CEA frameworks to strengthen LMICs' in-country institutional capacity for evidence-based policymaking.

The systematic reviews and meta-analyses described in Table 3 reported LDCT screening CEA with sources derived from HICs ${ }^{110-113}$. However, economic evaluations mainly are helpful at the country level if they are context-specific, and transferability from HICs may be inaccurate and misleading ${ }^{114,115}$. Decisions should consider local values regarding equity, population-specific data, local costs of interventions (for example, medical, non-medical and productivity losses), temporally dependent price points and informed local judgments of feasibility. Unfortunately, cancer screening cost-effectiveness literature in developing countries is scarce: a systematic review identified nine CEAs for breast cancer, two for colorectal and one for liver cancer prevention ${ }^{56}$. To our knowledge, there is no CEA of LDCT screening in LMICs yet available in the published English literature. Although one should not translate CEA from HICs to LMICs, aspects of research from HICs might still inform the analysis in other contexts, and extrapolation may be the first methodological step in assessing the need for further local and realistic studies ${ }^{106}$.

\section{Strategies to enable LDCT implementation in LMICs}

The strategies to increase LDCT efficacy, applicability and cost-effectiveness in LMICs are listed in Table 4 and are discussed here in more detail.
(1) Eligibility criteria. Risk-prediction models (RPMs) are used to refine screening eligibility and improve effectiveness while reducing resource utilization. Numerous RPMs have been developed ${ }^{116}$, demonstrating mortality benefit and lower NNS compared with the United States Prevention Services Task Force eligibility criteria $^{117}$. For instance, the $\mathrm{PLCO}_{\mathrm{m} 2012}$ model incorporates lung cancer personal and family history, as well as coexisting COPD, and was validated to recommend screening at a 6-year lung cancer risk cutoff of $>2 \%$, lowering the number of individuals screened in the NLST population by $81 \%$ (ref. ${ }^{118}$ ). The $\operatorname{LLP}_{\mathrm{v} 2}$ was assessed in the community-based Liverpool Healthy Lung Programme, using a cutoff of $\geq 5 \%$ risk over five years, with predicted results, though tentative, similar to those observed in the NLST (22\% versus $20 \%$ lung cancer mortality RR, respectively ${ }^{119}$. These multivariable RPMs are currently being validated in the National Health Services Targeted Lung Health Checks Programme, in England, at a proposed risk threshold $\geq 1.51 \%$ over six years and $\geq 2.5 \%$ over five years, respectively, for the $\operatorname{PLCO}_{\mathrm{m} 2012}$ and the $\operatorname{LLP}_{\mathrm{v} 2}{ }^{120}$, both of which were endorsed by the European Union position statement in 2017 (ref. ${ }^{121}$ ).

Inclusion criteria for screening have a major impact on cost-effectiveness $^{122}$. For example, micro-simulation models comparing the use of the Chinese guidelines with those of the US Centers of Medicare and Medicaid Services concluded that the application of local recommendations would result in 50\% improvement in mortality reduction in females (2.79\% versus $1.97 \%)$, saving an additional 20,000 lives in China by 2050, but only if almost $50 \%$ more screenings were performed ${ }^{123}$. In such cases, RPM utilization could optimize screening eligibility and effectiveness, possibly resulting in a substantial reduction in budget impact and making LDCT screening eventually cost-saving if non-curative treatment costs continue to rise ${ }^{97}$. An important consideration when calibrating RPMs is for individuals who will be left out and not benefit from screening ${ }^{117}$. Hence, the development and validation of RPMs and tools tailored to each LMIC perspective is genuinely needed to render program implementation effective, equitable and affordable ${ }^{123}$.

(2) Screening interval. Biennial screening is potentially as effective as annual screening, with no differences in interval lung cancer diagnosis, specificity and positive versus negative predictive values $^{28}$. Accordingly, the results of the NELSON trial regarding gradually spaced screening indicate that a 2 -year interval is safe and effective $^{27}$. A polynomial model for personalized follow-up intervals using both patient characteristics and baseline scan morphology has also been described as superior to other models ${ }^{124}$. Although this approach requires more robust validation ${ }^{125}$, biennial or tailored screening intervals according to the presence of nodules on baseline CT, along with risk assessment frameworks, could be applied in resource-restricted areas, potentially reducing LDCTs by about one third $^{126}$ and enhancing cost-effectiveness ${ }^{127}$.

(3) Smoking cessation. Tobacco control is the most important intervention in the fight against lung cancer. Abstinence for at least seven years results in a reduction in lung cancer mortality similar to that from LDCT screening in patients with $a \geq 30$-pack-per-year history $(20 \%)^{128}$. Fortunately, evidence-based tobacco control policies have achieved considerable progress in LMICs, which presently account for over $50 \%$ of the top-performing countries in that context ${ }^{129}$.

There is also a synergistic effect between the two interventions. Every additional year without smoking decreases lung cancer deaths by $9 \%$ with, and $6 \%$ without, screening, with similar benefits noted for overall mortality ${ }^{128}$. Therefore, smoking cessation interventions must be prioritized in LCS programs. Screening counseling should address potential false reassurance that negative results of LDCT screening might be an encouragement to continue smoking ${ }^{63}$ and 


\section{Table 3 | Cost-effectiveness analyses of lung cancer LDCT screening: systematic reviews and meta-analyses}

\begin{tabular}{l} 
Title \\
\hline Low-dose computed tomography \\
for lung cancer screening: \\
a review of the clinical \\
effectiveness, diagnostic \\
accuracy, cost-effectiveness and \\
guidelines \\
Cost-effectiveness of screening \\
for lung cancer with low-dose \\
computed tomography: a \\
systematic literature review
\end{tabular}

Cost-effectiveness analyses of lung cancer screening strategies using low-dose computed tomography: a systematic review

Low-dose computed tomography Snowsill et al. ${ }^{113}$ for lung cancer screening in high-risk populations: a systematic review and economic evaluation

\begin{tabular}{|c|c|c|}
\hline Author & Year & $\begin{array}{l}\text { Papers analyzed } \\
\text { (number) }\end{array}$ \\
\hline $\begin{array}{l}\text { Canadian Agency } \\
\text { for Drugs and } \\
\text { Technologies in } \\
\text { Health }\end{array}$ & 2015 & $\begin{array}{l}8 \text { studies published } \\
\text { from } 2012 \text { to } 2015 \text { : } \\
5 \text { from the US } \\
1 \text { from Canada } \\
1 \text { from Japan } \\
1 \text { from Israel }\end{array}$ \\
\hline Puggina et al..111 & 2015 & $\begin{array}{l}9 \text { studies published up } \\
\text { to } 31 \text { March 2015: } \\
7 \text { from the US } \\
1 \text { from Israel } \\
1 \text { from Australia }\end{array}$ \\
\hline Raymakers et al. ${ }^{112}$ & 2016 & $\begin{array}{l}13 \text { studies published } \\
\text { from } 2000 \text { to } 2014 \text { : } \\
10 \text { from the US } \\
1 \text { from Australia } \\
1 \text { from Israel } \\
1 \text { from Japan }\end{array}$ \\
\hline Snowsill et al. ${ }^{113}$ & 2018 & $\begin{array}{l}19 \text { studies published } \\
\text { from } 2001 \text { to } 2017 \text { : } \\
11 \text { from the US } \\
3 \text { from Canada } \\
2 \text { from the UK } \\
1 \text { from Israel } \\
1 \text { from Japan } \\
1 \text { from Australia }\end{array}$ \\
\hline
\end{tabular}

1 from Australia

\section{Conclusion}

Comparison between studies was difficult as assumptions and model parameters varied.
Cost-effectiveness of LDCT screening for lung cancer is highly debatable. Currently available economic evaluations suggest that LDCT for lung cancer screening is cost effective compared with no screening and indicate that the implementation of LDCT should be considered when planning a national lung cancer screening program. Additional economic evaluations, especially from a societal perspective and in an EU setting, are needed.

The cost-effectiveness of a lung cancer screening program using LDCT remains to be conclusively resolved. It is expected that this will largely depend on identifying an appropriate group of high-risk subjects.

LDCT screening may be clinically effective in reducing lung cancer mortality, but there is considerable uncertainty. There is evidence that a single round of screening could be considered cost effective at conventional thresholds, but there is significant uncertainty about the effect on costs and the magnitude of benefits.

Results were obtained by a search of PUBMED and EMBASE on 8 March 2020 with the search terms ('cost-effectiveness' or feasibility) and ('lung cancer' or NSCLC) and (LDCT or CT or 'low-dose computed tomography'); systematic reviews and meta-analyses in the English language that evaluated LDCT lung cancer screening cost-effectiveness and were published over the previous 10 years were included. EU, European Union;

should serve as an additional 'teachable moment' for smoking cessation $^{130}$. Successful results are endorsed by a subsequent analysis from the NLST, demonstrating that approximately $40 \%$ of individuals had quit smoking at the 7-year follow-up, compared with 5\% in the general population ${ }^{131}$. Yet an integrative standardized methodology is needed, as up to $90 \%$ of health professionals regularly ask about tobacco use, but less than half discuss medications or assist with smoking cessation ${ }^{128}$. Likely affordable strategies include integrating CHWs within the screening program, use of telephone helplines and web-based smoking cessation resources ${ }^{62}$.

Smoking control is listed as among the most beneficial interventions, at the lowest cost, by the WHO, being projected to cost around US $\$ 0.6$ billion for all LMICs combined, or US $\$ 0.11$ per capita annually ${ }^{132}$. In perspective, a tripling of the excise tax on tobacco could mobilize an extra US $\$ 100$ billion worldwide in annual revenue, which, in turn, could be invested in both primary and secondary prevention, including LDCT screening ${ }^{133}$. Given the substantial evidence that tobacco cessation policies are cost-effective per $\mathrm{se}^{2,134}$, simulation studies suggest that an approach combining these with LDCT is more cost-effective than LDCT alone, cutting the costs of screening in half ${ }^{135}$. To better characterize the benefits of both strategies in LMICs, further LCS implementation CEA must include trade-off analyses considering smoking cessation alone and combined with LDCT screening.

(4) Initial implementation. Implementation pilot studies and demonstration studies are vital, as RCTs generate evidence in an ideal environment that differs from the real-life situation. Implementation science research refers to the study of strategies and application of evidence to local health delivery settings. This approach can inform stakeholders about the applicability and economic feasibility of LDCT screening to their particular context, identify quality improvement areas and help with scale-up ${ }^{87}$. Seeking international consultations with previously established programs is also encouraged as an opportunity to share know-how involving the necessary steps for screening implementation and for planning for future integration into large health systems ${ }^{136}$. For instance, completed and ongoing community-based pilot programs from the United Kingdom have successfully engaged high-risk individuals of lower socioeconomic status and deprived communities with effective outcomes, and can serve as a reference for LMICs ${ }^{60,119,120,137}$. Finally, large-scale demonstration programs can help define and build new infrastructure, train healthcare workers and harness opportunities to overcome local resource limitations ${ }^{138}$. Stepwise implementation of LDCT screening that would initially focus in areas with higher lung cancer incidence and mortality rates is a reasonable option ${ }^{87}$, as demonstrated in China ${ }^{139}$. However, it is essential to note that the ultimate goal is equitable resource allocation, particularly in underprivileged communities.

(5) Monitoring lung cancer. Lung cancer incidence is under-reported in LMICs ${ }^{140}$. Currently, only one out of five LMICs generate the reliable population-based data necessary for cancer control $^{2}$. For instance, the Chinese National Cancer Registry contains information from roughly $13 \%$ of China's population, in contrast with $96 \%$ and $100 \%$ coverage in the United States and United Kingdom, respectively ${ }^{141}$. The creation and expansion of national cancer databases is urgently needed in LMICs, as the optimal 
Table 4 | Strategies to increase efficacy, applicability and cost-effectiveness of LDCT lung cancer screening in LMICs

\begin{tabular}{|c|c|c|}
\hline Screening process & Strategy & Desired impact \\
\hline Eligibility criteria & $\begin{array}{l}\text { Develop and validate tailored RPM balanced according to } \\
\text { local lung cancer epidemiology and screening capacity }\end{array}$ & $\begin{array}{l}\text { Optimization of screening benefit in high-risk participants } \\
\downarrow \text { LDCT utilization } \\
\uparrow \text { Cost-effectiveness }\end{array}$ \\
\hline Screening interval & Biennial or personalized ${ }^{a}$ & $\begin{array}{l}\downarrow \text { LDCT utilization } \\
\uparrow \text { Cost-effectiveness }\end{array}$ \\
\hline Smoking cessation & $\begin{array}{l}\text { Integrate tobacco control interventions within LCS } \\
\text { programs }^{\text {b }}\end{array}$ & $\begin{array}{l}\text { Addressing false reassurance of encouragement to smoke with } \\
\text { screening } \\
\text { Higher quit rates than with smoking cessation alone }{ }^{c} \\
\uparrow \text { Cost-effectiveness }\end{array}$ \\
\hline Initial implementation & $\begin{array}{l}\text { Consult previously established programs } \\
\text { Perform pilot studies with subsequent large-scale } \\
\text { demonstration studies }\end{array}$ & $\begin{array}{l}\text { Identification of areas of improvement } \\
\text { Development of new infrastructure and fostering of know-how } \\
\text { development before scaling up }\end{array}$ \\
\hline \multirow[t]{2}{*}{ Monitoring lung cancer } & Develop or expand national lung cancer databases & $\begin{array}{l}\text { Understanding of local lung cancer macrodeterminants } \\
\text { Optimization of resource allocation }\end{array}$ \\
\hline & Create screening registries & $\begin{array}{l}\text { Program quality monitoring } \\
\text { Investment evaluation }\end{array}$ \\
\hline \multirow[t]{2}{*}{ Infrastructural demand } & Implement public-private partnerships & $\begin{array}{l}\text { Use of idle capacity of private system } \\
\downarrow \text { Costs }\end{array}$ \\
\hline & Apply new technologies ${ }^{d}$ & $\begin{array}{l}\text { Overcoming local limitations } \\
\downarrow \text { Costs }\end{array}$ \\
\hline Payment & $\begin{array}{l}\text { Implement payment reform } \\
\text { Include new procurement and pricing schemes in screening } \\
\text { guidelines }\end{array}$ & $\begin{array}{l}\uparrow \text { Access to cancer care } \\
\uparrow \text { Affordability } \\
\uparrow \text { Cost-effectiveness }\end{array}$ \\
\hline
\end{tabular}

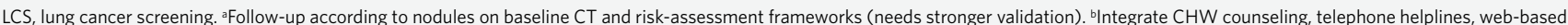
resources. ${ }^{4} 40 \%$ on NLST compared with $5 \%$ in the US general population. Institutional twinning and collaborations using telehealth, international collaborations via open-source networks.

ePay-per-performance, price discrimination, cost-sharing.

organization and distribution of healthcare resources rely on available information about gaps in equipment, treatment facilities, personnel and funding in specific regions ${ }^{136}$. It also enables a better understanding of how and why macroeconomic determinants influence lung cancer indicators locally. National databases are therefore key for successful LCS implementation and must be generated along with the establishment of the screening registries needed for post-intervention assessment and quality assurance mechanisms, as well as investment monitoring and evaluation. To address the scarcity of reliable data, the Global Initiative for Cancer Registry Development promotes cooperation of several international organizations with LMICs, and provides the technical and funding support necessary to foster cancer database development ${ }^{142}$.

(6) Public-private partnerships. In many LMICs, publicly funded healthcare coexists with a private system that usually serves the wealthier population. In India, the private sector expanded from $8 \%$ to $93 \%$ of all hospitals and $80 \%$ of outpatient visits between 1947 and 2011 (ref. ${ }^{143}$ ). In South Africa, there is a relatively small budget for the National Department of Health alongside a sizable private sector, accounting for more than $50 \%$ of expenses while servicing $20 \%$ of the population ${ }^{144}$. In such scenarios, a synergistic approach between public and private systems using properly regulated private infrastructure, experienced providers and idle equipment capacity could be contracted by publicly financed programs for lower budgets. The rise in private-sector demand would lower costs ${ }^{145}$ and public payer burden, ultimately increasing LCS feasibility. Notably, payment incentives should align with high quality and positive outcomes to avoid mismanagement of public resources ${ }^{56}$.

(7) Applying new technologies. The use of models of care enhanced by mobile phones and cloud applications is becoming more widely feasible in LMICs and has the potential to optimize participation and follow-up of abnormal screening results, as well as to improve patient-physician communication. Pairing district hospitals with central cancer centers through telemedicine, telepathology and continued medical education can promote protocol standardization, raise the quality of cancer care and lower costs ${ }^{56}$. For instance, ONCONET-India, a project created to enhance collaboration among regional cancer centers and peripheral medical centers, is estimated to reach over 300 hospitals country-wide ${ }^{59}$. Another initiative, the Early Lung Imaging Confederation pilot, provides an open-source environment to analyze extensive collections of LDCT images, supporting a global network to develop and validate imaging tools ${ }^{146}$. Ultimately, applying new technologies would decrease the need to employ local expertise or advanced equipment for many aspects of LCS in LMICs, as advanced communication and information technology would facilitate virtual collaboration.

(8) Payment. The underfinancing of cancer care is also known as the '5/80 disequilibrium', whereby which only $5 \%$ of global resources for cancer control are invested in LMICs despite $80 \%$ of disability-adjusted life-years lost coming from this population ${ }^{138}$. Furthermore, out-of-pocket costs paid by patients represent up to $50 \%$ of overall medical costs in LMICs, harming access to care and leading to catastrophic health expenditure estimated to push millions into poverty every year ${ }^{147}$. For instance, the price of a CT scan in Pakistan is estimated to be US\$99-1,000, the lower end of which represents more than a month's salary for most citizens ${ }^{63}$. Thus, stakeholders in LMICs need to leverage 'high-value oncology' by designing and expanding UHC with pricing schemes and pay-per-performance incentives that can help push the premise that any intervention costs should be linked to its benefit, bringing the LDCT screening program price closer to the country-specific thresholds in $\mathrm{LMICs}^{148}$. As an example, the Philippines' National Centre for Pharmaceutical Access and Management ${ }^{149}$ and Brazil's 
National Committee for Technology Incorporation ${ }^{150}$ provide help with designing payment schemes, performance measurements and pathways of care and setting the research agenda in concordance with national values and implementation feasibility.

LDCT screening guidelines should include new financing and procurement mechanisms tailored to resource-restricted areas ${ }^{98}$. For instance, charging different prices for a product in different markets, usually based on the ability to pay (i.e., price discrimination), and cost-sharing between the industry and private or public payer are used in many industries and are efficacious in LMICs ${ }^{151}$. In Egypt, a country-wide hepatitis $\mathrm{C}$ screening program effort, promoted by a national committee specially developed for the task, was successfully adopted in 2020, providing free screening to over 50 million people and reducing initial costs per treatment from US $\$ 84,000$ to US $\$ 45$. This program should serve as a benchmark for other LMICs, showing that dramatic improvement in public health is possible without enormous cost through social pressure, political will, mass procurement, smart financial and human resources allocation, and efficient information-technology support ${ }^{152}$.

\section{Conclusions}

This article is not a systematic review, and some of the data analyzed have limited power. Observational studies, RCTs or CEA assessing LDCT screening in LMICs are scarce, if not absent. Thus, we used the currently available information about general and cancer-specific health policies to discuss the practicability and affordability of implementing LCS in LMICs. Beyond the scope of this review, more nuanced discussions around pricing structures, reimbursement and broader macroeconomic determinants of lung cancer in LMICs are needed.

These limitations notwithstanding, the present discussion underscores the urgent need to devise optimal means for combating the growing burden of lung cancer in LMICs and reducing the disproportionate mortality rates these countries face. Without better prevention and new financing strategies, the increased encumbrance of lung cancer can make the treatment virtually unaffordable in the long term as the world now faces the enormous and unperceived cost of inaction. Investing in tobacco cessation, as the most cost-effective intervention, is paramount.

Moreover, the effectiveness, affordability, feasibility and cost-effectiveness of LDCT screening vary among LMICs, as different countries differ in lung cancer epidemiological patterns and the readiness of their healthcare systems, resulting in a need for country-specific analyses. Local priorities respected, we endorse the call to challenge the idea that LMICs cannot deliver high-quality cancer care ${ }^{138}$. Obstacles may be surmounted by tailored strategies and policies that are not merely based on HICs-derived guidelines.

Currently, the coronavirus disease 2019 (COVID-19) global pandemic threatens to undermine vulnerable health systems in LMICs and to negatively impact cancer diagnosis and care. Although different countries have mounted distinct responses with various levels of success, the overall implications of this pandemic are likely to aggravate the global and LMIC lung cancer burdens. The need to divert healthcare staff and resources, and the temporary halting of non-urgent surgery, radiotherapy and chemotherapy services to respond to the pandemic, are hampering cancer diagnoses, the provision of cancer care necessary for LCS and subsequent treatment in many countries. The ongoing economic downturn is expected to afflict LMICs most intensely and may thereby delay the implementation of LDCT screening programs. Stakeholders must carefully assess each country's economic conditions and healthcare capacity and devise urgent plans to address challenges relating to COVID-19 exposure and demand for health services so that patients with cancer receive appropriate care ${ }^{153}$.

This Review does not intend to lead to a common analysis of LDCT screening in LMICs, but rather to spur dialog within countries about this life-saving tool. Ultimately, we hope that such discussion will enable necessary research endeavors and steer the course of policy changes and, if context-appropriate, downstream rational and personalized national implementation plans.

Received: 7 August 2019; Accepted: 9 October 2020;

Published online: 30 November 2020

\section{References}

1. Bray, F. et al. Global cancer statistics 2018: GLOBOCAN estimates of incidence and mortality worldwide for 36 cancers in 185 countries. CA J. Clin. 68, 394-424 (2018).

2. Cleary, J., Gelband, H. \& Wagner, J. Cancer: Disease Control Priorities 3rd edn. (World Bank Group, 2015).

3. Kocher, F. et al. Longitudinal analysis of 2293 NSCLC patients: a comprehensive study from the TYROL registry. Lung Cancer 87, 193-200 (2015).

4. Goldstraw, P. et al. The IASLC Lung Cancer Staging Project: proposals for revision of the TNM stage groupings in the forthcoming (eighth) edition of the TNM Classification for Lung Cancer. J. Thoracic Oncol. 11, 39-51 (2016).

5. Kubík, A. \& Polák, J. Lung cancer detection. Results of a randomized prospective study in Czechoslovakia. Cancer 57, 2427-2437 (1986).

6. Berlin, N. I., Buncher, C. R., Fontana, R. S., Frost, J. K. \& Melamed, M. R. The National Cancer Institute Cooperative Early Lung Cancer Detection Program. Results of the initial screen (prevalence). Early lung cancer detection: Introduction. Am. Rev. Respir. Dis. 130, 545-549 (1984).

7. Aberle, D. R. et al. Reduced lung-cancer mortality with low-dose computed tomographic screening. N. Engl. J. Med. 365, 395-409 (2011).

8. de Koning, H. J. et al. Reduced lung-cancer mortality with volume CT screening in a randomized trial. N. Engl. J. Med. 382, 503-513 (2020).

9. Brett, G. Z. The value of lung cancer detection by six-monthly chest radiographs. Thorax 23, 414-420 (1968).

10. Oken, M. M. et al. Screening by chest radiograph and lung cancer mortality: the Prostate, Lung, Colorectal, and Ovarian (PLCO) randomized trial. J. Am. Med. Assoc. 306, 1865-1873 (2011).

11. Henschke, C. I. et al. Early Lung Cancer Action Project: overall design and findings from baseline screening. Lancet 354, 99-105 (1999).

12. Henschke, C. I. et al. Early Lung Cancer Action Project: initial findings on repeat screenings. Cancer 92, 153-159 (2001).

13. Henschke, C. I. et al. Survival of patients with stage I lung cancer detected on CT screening. N. Engl. J. Med. 355, 1763-1771 (2006).

14. Kaneko, M. et al. Peripheral lung cancer: screening and detection with low-dose spiral CT versus radiography. Radiology 201, 798-802 (1996).

15. Sone, S. et al. Mass screening for lung cancer with mobile spiral computed tomography scanner. Lancet 351, 1242-1245 (1998).

16. Wilson, D. O. et al. The Pittsburgh Lung Screening Study (PLuSS): outcomes within 3 years of a first computed tomography scan. Am. J. Respir. Crit. Care Med. 178, 956-961 (2008).

17. Menezes, R. J. et al. Lung cancer screening using low-dose computed tomography in at-risk individuals: the Toronto experience. Lung Cancer 67, 177-183 (2010).

18. Swensen, S. J. et al. CT screening for lung cancer: five-year prospective experience. Radiology 235, 259-265 (2005).

19. Sobue, T. et al. Screening for lung cancer with low-dose helical computed tomography: anti-lung cancer association project. J. Clin. Oncol. 20, 911-920 (2002).

20. Bach, P. B. et al. Benefits and harms of CT screening for lung cancer: a systematic review. J. Am. Med. Assoc. 307, 2418-2429 (2012).

21. Blanchon, T. et al. Baseline results of the Depiscan study: a French randomized pilot trial of lung cancer screening comparing low dose CT scan (LDCT) and chest X-ray (CXR). Lung Cancer 58, 50-58 (2007).

22. Paci, E. et al. Mortality, survival and incidence rates in the ITALUNG randomised lung cancer screening trial. Thorax 72, 825-831 (2017).

23. Infante, $M$. et al. Long-term follow-up results of the DANTE trial, a randomized study of lung cancer screening with spiral computed tomography. Am. J. Respir. Crit. Care Med. 191, 1166-1175 (2015).

24. Becker, N. et al. Lung cancer mortality reduction by LDCT screeningresults from the randomized German LUSI trial. Int. J. Cancer 146 1503-1513 (2020)

25. Wille, M. M. et al. Results of the randomized Danish Lung Cancer Screening Trial with focus on high-risk profiling. Am. J. Respir. Crit. Care Med. 193, 542-551 (2016).

26. Doroudi, M., Pinsky, P. F. \& Marcus, P. M. Lung cancer mortality in the Lung Screening Study Feasibility Trial. JNCI Cancer Spectr. 2, pky042 (2018)

27. Duffy, S. W. \& Field, J. K. Mortality reduction with low-dose CT screening for lung cancer. N. Engl. J. Med. 382, 572-573 (2020).

28. Pastorino, U. et al. Prolonged lung cancer screening reduced 10-year mortality in the MILD trial: new confirmation of lung cancer screening efficacy. Ann. Oncol. 30, 1162-1169 (2019). 
29. Office on Smoking and Health (US Centers for Disease Control and Prevention /National Center for Chronic Disease Prevention and Health Promotion). How Tobacco Smoke Causes Disease: The Biology and Behavioral Basis for Smoking-Attributable Disease: A Report of the Surgeon General https://www.ncbi.nlm.nih.gov/books/NBK53017/ (CDC, Atlanta, 2010).

30. Jha, P. Avoidable global cancer deaths and total deaths from smoking. Nat. Rev. Cancer 9, 655-664 (2009).

31. World Health Organization. WHO Report on the Global Tobacco Epidemic 2019 https://apps.who.int/iris/bitstream/handle/10665/326043/97892415162 04-eng.pdf?ua=1 (WHO, 2019).

32. Cheng, T. Y. D. et al. The international epidemiology of lung cancer: latest trends, disparities, and tumor characteristics. J. Thorac. Oncol. 11, 1653-1671 (2016)

33. Couraud, S., Zalcman, G., Milleron, B., Morin, F. \& Souquet, P. J. Lung cancer in never smokers-a review. Eur. J. Cancer 48, 1299-1311 (2012).

34. Sisti, J. \& Boffetta, P. What proportion of lung cancer in never-smokers can be attributed to known risk factors? Int. J. Cancer 131, 265-275 (2012).

35. Islami, F. et al. Cancer deaths and cases attributable to lifestyle factors and infections in China, 2013. Ann. Oncol. 28, 2567-2574 (2017).

36. Islami, F. et al. Proportion and number of cancer cases and deaths attributable to potentially modifiable risk factors in the United States. CA Cancer J. Clin. 68, 31-54 (2018).

37. Zhao, P., Dai, M., Chen, W. \& Li, N. Cancer trends in China. Jpn. J. Clin. Oncol. 40, 281-285 (2010).

38. Huang, C.-Y. et al. Unfavorable mortality-to-incidence ratio of lung cancer is associated with health care disparity. Int. J. Environ. Res. Public Health 15 2889 (2018).

39. Raez, L. E. et al. The burden of lung cancer in Latin-America and challenges in the access to genomic profiling, immunotherapy and targeted treatments. Lung Cancer 119, 7-13 (2018).

40. Raez, L. E. et al. Challenges in lung cancer screening in Latin America. J. Glob. Oncol. 4, 1-10 (2018).

41. Raghava, S. \& Siddque, S. PUB075: Survival of patients with stage I lung cancer detected on CT screening in South Indian population. J. Thorac. Oncol. 12, S1491-S1492 (2017).

42. Liu, X. et al. The outcome differences of CT screening for lung cancer pre and post following an algorithm in Zhuhai, China. Lung Cancer 73, 230-236 (2011).

43. Zhou, Q. et al. Demonstration program of population-based lung cancer screening in China: rationale and study design. Thorac. Cancer $\mathbf{5}$, 197-203 (2014).

44. Zhou, Q. MS16.02: NELCIN B3 screening program in China. J. Thorac. Oncol. 13, S272-S273 (2018)

45. Dai, M. S. J. \& Li, N. Design and goal of urban cancer early diagnosis and treatment project in China. Chin. J. Prev. Med. 47, 179-182 (2013).

46. Cheng, Y. I., Davies, M. P. A., Liu, D., Li, W. \& Field, J. K. Implementation planning for lung cancer screening in China. Precision Clinical Medicine 2, 13-44 (2019).

47. Wang, Z. et al. Mortality outcomes of low-dose computed tomography screening for lung cancer in urban China: a decision analysis and implications for practice. Chin. J. Cancer 36, 57 (2017).

48. Zhou, Q. et al. [China National Lung Cancer Screening Guideline with Low-dose Computed Tomography (2018 version)]. Zhongguo Fei Ai Za Zhi 21, 67-75 (2018)

49. Nikolaev, A. et al. [Three clinically relevant findings in lung cancer screening.]. Tuberculosis Lung. Dis. 97, 37-44 (2019).

50. UMCG. Important grant for collaboration in research on early detection of lung cancer, COPD and cardiovascular diseases. https://www.rug.nl/news/ 2016/11/grote-subsidie-voor-samenwerking-in-onderzoek-vroegeopsporing-longkanker-copd-en-hart--en-vaatziekten?lang=en\%3E (2016)

51. dos Santos, R. S. et al. Do current lung cancer screening guidelines apply for populations with high prevalence of granulomatous disease? Results from the First Brazilian Lung Cancer Screening Trial (BRELT1). Ann. Thorac. Surg. 101, 481-486 (2016).

52. Triphuridet, N., Singharuksa, S. \& Vidhyakorn, S. P1.03-043 Practical difficulty of low dose computerized tomography as a lung cancer screening tool in an endemic area of tuberculosis: topic: screening. J. Thorac. Oncol. 12, S568-S569 (2017).

53. Koegelenberg, C. F. N. et al. Recommendations for lung cancer screening in Southern Africa. J. Thorac. Dis. 11, 3696-3703 (2019).

54. Wood, D. E. et al. Lung Cancer Screening, Version 3.2018, NCCN Clinical Practice Guidelines in Oncology. J. Natl. Compr. Canc. Netw. 16, 412-441 (2018).

55. Moyer, V. A. Screening for lung cancer: U.S. Preventive Services Task Force recommendation statement. Ann. Intern. Med. 160, 330-338 (2014).

56. Gelband, H. et al. Costs, affordability, and feasibility of an essential package of cancer control interventions in low-income and middle-income countries: key messages from Disease Control Priorities, 3rd edition. Lancet 387, 2133-2144 (2016)
57. Pedersen, J. H. \& Ashraf, H. Implementation and organization of lung cancer screening. Ann. Transl. Med. 4, 152 (2016).

58. Li, J., Shi, L., Liang, H., Ding, G. \& Xu, L. Urban-rural disparities in health care utilization among Chinese adults from 1993 to 2011. BMC Health Serv. Res. 18, 102 (2018).

59. Goss, P. E. et al. Challenges to effective cancer control in China, India, and Russia. Lancet Oncol. 15, 489-538 (2014).

60. Crosbie, P. A. et al. Implementing lung cancer screening: baseline results from a community-based 'Lung Health Check' pilot in deprived areas of Manchester. Thorax 74, 405-409 (2019).

61. Lee, C. Screening for lung cancer: effective recruitment methods. AJR Am. J. Roentgenol. 210, 514-517 (2018).

62. Goulart, B. H. The value of lung cancer CT screening: it is all about implementation. Am. Soc. Clin. Oncol. Educ. Book 35, e426-e433 (2015).

63. Abbasi, A. et al. Prevalence and barriers to lung cancer screening in Karachi, Pakistan: a cross-sectional survey of smokers and physicians. Cureus 9, e1248 (2017).

64. Mohamed-Hussein, A. \& Ibrahim, M.-E. Evaluation of lung cancer screening practices of chest physicians in Egypt: a pilot national survey. Chest 146, 603A (2014).

65. Nhung, B. C. et al. Intentions to undergo lung cancer screening among Korean men. Asian Pac. J. Cancer Prev. 16, 6293-6298 (2015).

66. Raz, D. J. et al. Augmented meaningful use criteria to identify patients eligible for lung cancer screening. Ann. Thorac. Surg. 98, 996-1002 (2014).

67. Shastri, S. S. et al. Effect of VIA screening by primary health workers: randomized controlled study in Mumbai, India. J. Natl. Cancer Inst. 106, dju009 (2014)

68. Joshi, R. et al. Task shifting for non-communicable disease management in low and middle income countries-a systematic review. PLOS ONE 9, e103754 (2014).

69. Chapple, A., Ziebland, S. \& McPherson, A. Stigma, shame, and blame experienced by patients with lung cancer: qualitative study. Br. Med. J. 328, 1470 (2004).

70. Neal, C. B. E. et al. Cancer Stigma and Silence Around the World: A LIVESTRONG Report https://www.livestrong.org/sites/default/files/ what-we-do/reports/lsglobalresearchreport.pdf (2010).

71. Daher, M. Cultural beliefs and values in cancer patients. Ann. Oncol. 23(Suppl 3), 66-69 (2012).

72. Dikshit, R. et al. Cancer mortality in India: a nationally representative survey. Lancet 379, 1807-1816 (2012).

73. Basu, P. et al. Women's perceptions and social barriers determine compliance to cervical screening: results from a population based study in India. Cancer Detect. Prev. 30, 369-374 (2006).

74. Hou, S. I., Sealy, D. A. \& Kabiru, C. W. Closing the disparity gap: cancer screening interventions among Asians-a systematic literature review. Asian Pac. J. Cancer Prev. 12, 3133-3139 (2011).

75. Dinshaw, K. et al. Determinants of compliance in a cluster randomised controlled trial on screening of breast and cervix cancer in Mumbai, India. 1. Compliance to screening. Oncology 73, 145-153 (2007).

76. Tanner, N. T. \& Silvestri, G. A. Shared decision-making and lung cancer screening: let's get the conversation started. Chest 155, 21-24 (2019).

77. Organization for Economic Growth and Development. Organization for Economic Growth and Development (OECD) Statistics https://stats.oecd. org (2019).

78. MacMahon, $\mathrm{H}$. et al. Guidelines for management of incidental pulmonary nodules detected on CT images: from the Fleischner Society 2017. Radiology 284, 228-243 (2017).

79. Henschke, C. International Early Lung Cancer Action Program: Screening Protocol http://www.ielcap.org/sites/default/files/I-ELCAP-protocol.pdf (2016.).

80. Patz, E. F. et al. Overdiagnosis in low-dose computed tomography screening for lung cancer. JAMA Intern. Med. 174, 269-274 (2014).

81. Bai, C. et al. Evaluation of pulmonary nodules: clinical practice consensus guidelines for Asia. Chest 150, 877-893 (2016).

82. Mendez, L. C., Moraes, F. Y., Fernandes, G. D. S. \& Weltman, E. Cancer deaths due to lack of universal access to radiotherapy in the Brazilian Public Health System. Clin. Oncol. (R Coll Radiol) 30, e29-e36 (2018).

83. Gyawali, B., Bouche, G., Crisp, N. \& André, N. Challenges and opportunities for cancer clinical trials in low- and middle-income countries. Nat. Cancer 1, 142-145 (2020).

84. Noronha, V. et al. A fresh look at oncology facts on south central Asia and SAARC countries. South Asian J. Cancer 1, 1-4 (2012).

85. Yano, E. M. et al. Implementation and spread of interventions into the multilevel context of routine practice and policy: implications for the cancer care continuum. J. Natl. Cancer Inst. Monogr. 2012, 86-99 (2012).

86. Taplin, S. H. et al. Introduction: Understanding and influencing multilevel factors across the cancer care continuum. J. Natl. Cancer Inst. Monogr. 2012, 2-10 (2012) 
87. World Health Organization. World Health Organization National Cancer Control Programmes (NCCP). https://www.who.int/cancer/nccp/en/ (accessed 7 April 2020).

88. World Bank. World Bank 2019-2020 Income Classification. https:// datahelpdesk.worldbank.org/knowledgebase/articles/906519-world-bankcountry-and-lending-groups (accessed 19 March 2020).

89. Agan, B. K. \& Marconi, V. C. Non-communicable diseases: yet another challenge for HIV treatment and care in Sub-Saharan Africa. Clin. Infect. Dis. 71, 1874-1876 (2020).

90. Islamic Republic of Afghanistan. [A Basic Package of Health Services for Afghanistan.] http://moph.gov.af/Content/Media/Documents/BPHS2005-FINAL29122010162945969.pdf (15 November 2005).

91. Parkin, D. M., Bray, F., Ferlay, J. \& Jemal, A. Cancer in Africa 2012. Cancer Epidemiol. Biomarkers Prev. 23, 953-966 (2014).

92. Almeida Ribeiro, A. S. M. \& Barradas, R. Condições de Saúde da população brasileira. in Politica e Sistemas de Saúde no Brasil (eds. Giovanella, L. et al.) pp. 143-181 (Fiocruz and Cebes, 2012).

93. Yang, G. et al. Rapid health transition in China, 1990-2010: findings from the Global Burden of Disease Study 2010. Lancet 381, 1987-2015 (2013).

94. The Economist Intelligence Unit. Breakaway: the global burden of cancer-challenges and opportunities. http://graphics.eiu.com/marketing/ pdf/EIU_LIVESTRONG_Global_Cancer_Burden.pdf (accessed 2 January 2020).

95. Aguiar, P. et al. Cost-effectiveness and budget impact of lung cancer immunotherapy in South America: strategies to improve access. Immunotherapy 10, 887-897 (2018).

96. Arroyo-Hernández, M., Zinser-Sierra, J. W. \& Vázquez-García, J. C. [Lung-cancer screening program in Mexico]. Salud Publica Mex. 61, 347-351 (2019).

97. Cressman, S. et al. The cost-effectiveness of high-risk lung cancer screening and drivers of program efficiency. J. Thorac. Oncol. 12, 1210-1222 (2017).

98. Chalkidou, K. et al. Evidence-informed frameworks for cost-effective cancer care and prevention in low, middle, and high-income countries. Lancet Oncol. 15, e119-e131 (2014).

99. Bloom, D. E. et al. The Global Economic Burden of Non-communicable Diseases (World Economic Forum, 2011).

100. Gøtzsche, P. C. \& Jørgensen, K. J.. Screening for breast cancer with mammography. Cochrane Database Syst. Rev. 2013(6), CD001877 (2013).

101. US Food and Drug Administration. MQSA National Statistics http://www. fda.gov/Radiation-EmittingProducts/MammographyQualityStandards ActandProgram/FacilityScorecard/ucm113858.htm (2020).

102. Jemal, A. \& Fedewa, S. A. Lung cancer screening with low-dose computed tomography in the United States: 2010 to 2015. JAMA Oncol. 3, 1278-1281 (2017).

103. Stout, N. K. et al. Retrospective cost-effectiveness analysis of screening mammography. J. Natl. Cancer Inst. 98, 774-782 (2006).

104. Stout, N. K. et al. Benefits, harms, and costs for breast cancer screening after US implementation of digital mammography. J. Natl. Cancer Inst. 106, dju092 (2014).

105. Chalkidou, K. et al. Priority-setting for achieving universal health coverage. Bull. World Health Organ. 94, 462-467 (2016).

106. Wilkinson, T. et al. The International Decision Support Initiative reference case for economic evaluation: an aid to thought. Value Health 19, 921-928 (2016).

107. Baltussen, R. et al. Priority setting for universal health coverage: we need evidence-informed deliberative processes, not just more evidence on cost-effectiveness. Int. J. Health Policy Manag. 5, 615-618 (2016).

108. Dye, C. et al. The World Health Report 2013: Research for Universal Health Coverage. https://www.who.int/whr/2013/report/en/ (2013).

109. Netherlands Initiative for Capacity Development in Higher Education (NICHE). REVISE 2020: REthinking the Valuation of Interventions to improve priority SEtting. http://www.niche1.nl/projects/id=34title= revise_2020_rethinking_the_valuation_of_interventions_to_improve_ priority_setting (2016).

110. Canadian Agency for Drugs and Technologies in Health. CADTH Rapid Response Reports: Low-Dose Computed Tomography for Lung Cancer Screening: A Review of the Clinical Effectiveness, Diagnostic Accuracy, Cost-Effectiveness, and Guidelines (Canadian Agency for Drugs and Technologies in Health, 2015).

111. Puggina, A., Broumas, A., Ricciardi, W. \& Boccia, S. Cost-effectiveness of screening for lung cancer with low-dose computed tomography: a systematic literature review. Eur. J. Public Health 26, 168-175 (2016).

112. Raymakers, A. J. N. et al. Cost-effectiveness analyses of lung cancer screening strategies using low-dose computed tomography: a systematic review. Appl. Health Econ. Health Policy 14, 409-418 (2016).

113. Snowsill, T. et al. Low-dose computed tomography for lung cancer screening in high-risk populations: a systematic review and economic evaluation. Health Technol. Assess. 22, 1-276 (2018)
114. Sculpher, M. J.et al. Generalisability in economic evaluation studies in healthcare: a review and case studies. Health Technol. Assess. 8iii-iv, 1-192, https://doi.org/10.3310/hta8490 (2004).

115. Murray, C. J., Evans, D. B., Acharya, A. \& Baltussen, R. M. Development of WHO guidelines on generalized cost-effectiveness analysis. Health Econ. 9, 235-251 (2000).

116. Katki, H. A. et al. Implications of nine risk prediction models for selecting ever-smokers for computed tomography lung cancer screening. Ann. Intern. Med. 169, 10-19 (2018)

117. Katki, H. A., Kovalchik, S. A., Berg, C. D., Cheung, L. C. \& Chaturvedi, A. K. Development and validation of risk models to select ever-smokers for ct lung cancer screening. J. Am. Med. Assoc. 315, 2300-2311 (2016).

118. Tammemagi, M. C. et al. Selection criteria for lung-cancer screening. $N$. Engl. J. Med. 368, 728-736 (2013).

119. Ghimire, B. et al. Evaluation of a health service adopting proactive approach to reduce high risk of lung cancer: the Liverpool Healthy Lung Programme. Lung Cancer 134, 66-71 (2019).

120. National Health Service (UK). Targeted Screening for Lung Cancer with Low Radiation Dose Computer Tomography. https://www.england.nhs.uk/ wp-content/uploads/2019/02/targeted-lung-health-checks-standardprotocol-v1.pdf (NHS, 2019).

121. Oudkerk, M. et al. European position statement on lung cancer screening. Lancet Oncol. 18, e754-e766 (2017).

122. Field, J. K. et al. The UK Lung Cancer Screening Trial: a pilot randomised controlled trial of low-dose computed tomography screening for the early detection of lung cancer. Health Technol. Assess. 20, 1-146 (2016).

123. Sheehan, D. F., Criss, S. D., Gazelle, G. S., Pandharipande, P. V. \& Kong, C. Y. Evaluating lung cancer screening in China: implications for eligibility criteria design from a microsimulation modeling approach. PLoS ONE 12, e0173119 (2017).

124. Schreuder, A. et al. Lung cancer risk to personalise annual and biennial follow-up computed tomography screening. Thorax 73, 626-633 (2018).

125. Field, J. K. \& Duffy, S. W. Lung cancer CT screening: are we ready to consider screening biennially in a subgroup of low-risk individuals? Thorax 73, 1006-1007 (2018)

126. Sverzellati, N. et al. Low-dose computed tomography for lung cancer screening: comparison of performance between annual and biennial screen Eur. Radiol. 26, 3821-3829 (2016).

127. Goffin, J. R. et al. Biennial lung cancer screening in Canada with smoking cessation-outcomes and cost-effectiveness. Lung Cancer 101, 98-103 (2016).

128. Tanner, N. T. et al. The association between smoking abstinence and mortality in the National Lung Screening Trial. Am. J. Respir. Crit. Care Med. 193, 534-541 (2016).

129. World Health Organization. Report On The Global Tobacco Epidemic, 2017: Monitoring Tobacco Use and Prevention Policies. http://www.who.int/ tobacco/global_report/2017/en/ (WHO, 2017).

130. Villanti, A. C., Jiang, Y., Abrams, D. B. \& Pyenson, B. S. A cost-utility analysis of lung cancer screening and the additional benefits of incorporating smoking cessation interventions. PLoS One 8, e71379 (2013).

131. Tammemägi, M. C., Berg, C. D., Riley, T. L., Cunningham, C. R. \& Taylor, K. L. Impact of lung cancer screening results on smoking cessation. J. Natl. Cancer Inst. 106, dju084 (2014).

132. WHO. Scaling Up Action Against Non-communicable Diseases: How Much Will It Cost? https://www.who.int/nmh/publications/cost_of_inaction/en/ (WHO, 2011)

133. Jha, P. \& Peto, R. Global effects of smoking, of quitting, and of taxing tobacco. N. Engl. J. Med. 370, 60-68 (2014).

134. Cromwell, J., Bartosch, W. J., Fiore, M. C., Hasselblad, V. \& Baker, T. Cost-effectiveness of the clinical practice recommendations in the AHCPR guideline for smoking cessation. J. Am. Med. Assoc. 278, 1759-1766 (1997).

135. McMahon, P. M. et al. Cost-effectiveness of computed tomography screening for lung cancer in the United States. J. Thorac. Oncol. 6, 1841-1848 (2011)

136. Sivaram, S. et al. Population-based cancer screening programmes in low-income and middle-income countries: regional consultation of the International Cancer Screening Network in India. Lancet Oncol. 19, e113-e122 (2018).

137. Crosbie, P. A. et al. Yorkshire Lung Screening Trial (YLST): protocol for a randomised controlled trial to evaluate invitation to community-based low-dose CT screening for lung cancer versus usual care in a targeted population at risk. BMJ Open 10, e037075 (2020).

138. Farmer, P. et al. Expansion of cancer care and control in countries of low and middle income: a call to action. Lancet 376, 1186-1193 (2010).

139. Wang, H. et al. Lung cancer screening with low-dose CT in China: study design and baseline results from the first round screening arm. J. Thorac. Oncol. 12, S581-S582 (2017).

140. Sartorius, B. \& Sartorius, K. How much incident lung cancer was missed globally in 2012? An ecological country-level study. Geospat. Health 11, 396 (2016). 
141. National Cancer Center and Disease Prevention and Control Bureau, Ministry of Health. Chinese Cancer Registry Annual Report, 2012 (Military Medical Sciences Press (China), 2012).

142. World Health Organization. Global Initiative Cancer Research https://gicr. iarc.fr/building-capacity/ (accessed 10 August 2020).

143. Planning Commission of India. High Level Expert Group (HLEG) Report on Universal Health Coverage for India (Planning Commission of India, 2011).

144. Li, R., Hernandez-Villafuerte, K., Towse, A., Vlad, I. \& Chalkidou, K. Mapping priority setting in health in 17 countries across Asia, Latin America, and sub-Saharan Africa. Health Syst. Reform 2, 71-83 (2016).

145. Manser, R. et al. Cost-effectiveness analysis of screening for lung cancer with low dose spiral CT (computed tomography) in the Australian setting. Lung Cancer 48, 171-185 (2005).

146. Mulshine, J. L. et al. The International Association for the Study of Lung Cancer Early Lung Imaging Confederation. JCO Clin. Cancer Inform. 4, 89-99 (2020).

147. Yang, S. C. et al. Cost-effectiveness of implementing computed tomography screening for lung cancer in Taiwan. Lung Cancer 108, 183-191 (2017).

148. Lopes, GdeL. Jr., de Souza, J. A. \& Barrios, C. Access to cancer medications in low- and middle-income countries. Nat. Rev. Clin. Oncol. 10, 314-322 (2013).

149. Philippines National Center for Pharmaceutical Access and Management (NCPAM). https://rb.gy/fglv8l (accessed 18 March 2020).

150. Pereira, V. C., Barreto, J. O. M. \& Neves, F. A. D. R. Health technology reassessment in the Brazilian public health system: analysis of the current status. PLoS ONE 14, e0220131 (2019).

151. Gorokhovich, L. C. K. \& Shankar, R. J. Improving access to innovative medicines in emerging markets: evidence and diplomacy as alternatives to the unsustainable status quo. J. Health Diplom. 1, 1-19 (2013).
152. Waked, I. et al. Screening and treatment program to eliminate hepatitis C in Egypt. N. Engl. J. Med. 382, 1166-1174 (2020).

153. Richards, M., Anderson, M., Carter, P., Ebert, B. L. \& Mossialos, E. The impact of the COVID-19 pandemic on cancer care. Nat. Cancer $\mathbf{1}$, 1-3 (2020).

\section{Author contributions}

Conception and design, E.E.S., R.B.G., M.E.S., L.L.S., G.F.P.A., R.M.K.M. and G.L.; collection assembly of data, E.E.S., R.B.G., M.E.S., L.L.S., G.F.P.A., R.M.K.M. and G.L.; data analysis and interpretation, E.E.S., R.B.G., M.E.S., L.L.S. and G.L.; manuscript writing, E.E.S., R.B.G., L.L.d.S., M.E.S., G.F.P.A. and G.L.; tables and figures, E.E.S., M.E.S., L.L.d.S., G.F.P.A., G.L.; and final approval, E.E.S., R.B.G., M.E.S., L.L.d.S., G.F.P.A., R.M.K.M. and G.L.

\section{Competing interests}

The authors declare no competing interests.

\section{Additional information}

Supplementary information is available for this paper at https://doi.org/10.1038/ s43018-020-00142-z.

Correspondence should be addressed to E.E.

Reprints and permissions information is available at www.nature.com/reprints.

Publisher's note Springer Nature remains neutral with regard to jurisdictional claims in published maps and institutional affiliations.

(c) Springer Nature America, Inc. 2020 